\title{
INTERAÇÃO DE METRIBUZIN E NITROGÊNIO NO CULTIVO DA CENOURA
}

\section{INTERACTION OF METRIBUZIN AND NITROGEN IN CARROT CROP}

Felipe Oliveira Xavier ${ }^{\mathrm{a}}$, Deivide Patrik Alves ${ }^{\mathrm{a}}$, Laís Franchini Pucci ${ }^{\mathrm{a}}$, Gustavo Antônio Mendes Pereira ${ }^{\mathrm{a} *}$, Leonardo Angelo de Aquino ${ }^{\mathrm{a}}$, Marcelo Rodrigues dos Reis ${ }^{\mathrm{a}}$

${ }^{\mathrm{a} D e p a r t a m e n t o ~ d e ~ A g r o n o m i a, ~ U n i v e r s i d a d e ~ F e d e r a l ~ d e ~ V i c ̧ o s a, ~ M i n a s ~ G e r a i s, ~ B r a s i l . ~}$

*Autor correspondente: gustavogamp@hotmail.com.

\section{INFORMAÇÕES DO ARTIGO}

\section{Histórico do artigo:}

Recebido: 29 Abril 2020.

Aceito: 16 Julho 2020.

Publicado: 03 Agosto 2020.

\section{Palavras-chave/Keywords:}

Daucus carota L./ Daucus carota L.. Adubação nitrogenada/ Nitrogen fertilization.

Herbicida/ Herbicide.

Partição de assimilados/ Partition of assimilates.

\section{Financiamento:} CAPES, CNPq.

Direito Autoral: Este é um artigo de acesso aberto distribuído sob os termos da Licença Creative Commons, que permite uso, distribuição e reprodução irrestritos em qualquer meio, desde que $\mathrm{o}$ autor e a fonte originais sejam creditados.

\section{Citação deste artigo:}

XAVIER, F. O.; ALVES, D. P.; PUCCI, L. F.; PEREIRA, G. A. M.; AQUINO, L. A.; REIS, M. R. Interação de metribuzin e nitrogênio no cultivo da cenoura. Revista Brasileira de Herbicidas, v. 19, n. 1. 2020.

\begin{abstract}
RESUMO
O nitrogênio $(\mathrm{N})$ é um dos nutrientes mais extraídos no cultivo de cenoura. No entanto, altas doses de $\mathrm{N}$ pode favorecer o crescimento da parte aérea em relação a raiz da planta. A aplicação do metribuzin pode alterar a partição da biomassa em favorecimento das raízes. Desta forma, objetivou-se com esta pesquisa avaliar o efeito da aplicação de metribuzin em pós-emergência, e sua interação com nitrogênio sobre o crescimento axial e radial e produtividade das raízes de cenoura. Três experimentos foram conduzidos em campo, em distintos locais de cultivo. O delineamento experimental utilizado foi blocos casualizados, com quatro repetições, dispostos em esquema fatorial $2 \times 3$, composto por duas doses de metribuzin $\left(0\right.$ e $\left.432 \mathrm{~g}^{\text {i.a. }} \mathrm{ha}^{-1}\right)$ e três doses de $\mathrm{N}$ em cobertura $\left(0,30 \mathrm{e} 90 \mathrm{~kg} \mathrm{ha}^{-1}\right)$. Avaliouse o comprimento e diâmetro de raízes, massa da matéria seca da parte aérea e raiz das plantas e, a produtividade de raízes. Não houve interação entre o metribuzin e nitrogênio nas variáveis estudadas. No entanto, a aplicação de metribuzin reduziu a massa da matéria seca da raiz da cenoura devido redução do diâmetro, sem ocasionar redução da produtividade. A aplicação do metribuzin associado à fertilização de $\mathrm{N}$ em cobertura não influencia no crescimento axial ou radial da raiz da cenoura e, consequentemente, não altera a produtividade.
\end{abstract}




\section{Introdução}

O nitrogênio (N) é um dos principais nutrientes exigidos no cultivo de cenoura (Daucus carota L.), influenciando diretamente na produtividade da cultura. Para que a cultura alcance produtividade próxima à $80 \mathrm{t} \mathrm{ha}^{-1} \mathrm{de}$ raízes é necessária a extração de $163 \mathrm{~kg} \mathrm{ha}^{-1}$ de $\mathrm{N}$ (DEZORDI et al., 2015). Assim, torna-se necessário aumentar as doses de $\mathrm{N}$ usualmente empregadas na cultura. No entanto, o aumento da fertilização de $\mathrm{N}$, deve ser realizada com cautela, pois pode acarretar em incrementos limitados na produtividade de raízes, relacionados com o direcionamento para a produção de parte aérea (OLIVEIRA, 2015).

Nos cultivos de verão, devido às altas temperaturas, em plantas de cenoura há maior produção de biomassa da parte aérea da planta em detrimento das raízes (OLIVEIRA, 2015). Assim, o coeficiente de utilização biológico (CUB) deste nutriente é reduzido no verão, com pouco acréscimo em produtividade de raízes por unidade de nutriente acumulado na planta (DEZORDI et al., 2015). Além disto, o aumento na produção da parte aérea da planta pode aumentar o período de irrigação necessário à cultura, tornando os custos de produção maiores.

A utilização do metribuzin em pós emergência na cultura da cenoura para o controle de plantas daninhas, além de ser uma boa opção pela alta eficácia de controle (JENSEN; DOOHAN; SPECHT, 2004), pode modificar alguns processos fisiológicos na planta, e afetar a partição da biomassa em favorecimento das raízes. Carneiro (2016) verificou menor produção de massa da matéria seca da parte aérea da cenoura com a aplicação de $576 \mathrm{~g}$ i.a. ha ${ }^{-1}$ de metribuzin aos 30 dias após a aplicação. Assim, o uso deste herbicida pode reduzir a taxa de velocidade de crescimento da parte aérea e estimular o crescimento das raízes, possibilitando aumentar as doses de $\mathrm{N}$ empregadas e, consequentemente, aumentar a produtividade.

O metribuzin é um herbicida pertencente ao grupo dos inibidores do fotossistema II (FS II), que quando presente em plantas sensíveis se liga a proteína D1, impedindo assim o fluxo de elétrons, com consequências na paralização da produção de ATP e $\mathrm{NADPH}_{2}$, além da produção de espécies reativas de oxigênio, que desencadeiam uma serie de reações que promovem a perioxidação da membrana celular (SILVA; SILVA, 2013).

São escassos na literatura trabalhos que relacionam o uso de herbicidas inibidores do FS II como reguladores de crescimento e a adubação nitrogenada, especialmente para as olerícolas. Desta forma, objetivou-se com este trabalho avaliar o efeito da aplicação de metribuzin em pósemergência, e sua interação com o nitrogênio sobre $o$ crescimento axial e radial e produtividade das raízes de cenoura.

\section{Material e Métodos}

Três experimentos foram conduzidos no campo, em distintos locais de cultivo da cenoura do município de Rio Paranaíba, MG. O primeiro experimento foi conduzido em talhão comercial - local $\mathrm{A}$, instalado no período de transição outono-inverno, entre março e julho de 2016, semeando-se a cultivar Verano, em semeadura direta, população de $650 \mathrm{mil}$ plantas, canteiro com 3 linhas duplas de cenoura com largura de 1,75 metros. O segundo experimento, conduzido no período de inverno, entre junho e setembro de 2016, com a cultivar Maestro - local B. O terceiro experimento foi conduzido no período de inverno, entre abril e agosto de 2017, com a cultivar Natuna - local C. Os solos de todos os locais foram classificados como Latossolo VermelhoAmarelo, de textura argilosa e corrigidos de acordo com a análise química (Tabela 1). Os dados meteorológicos dos três experimentos estão demonstrados na figura 1 .

O delineamento experimental utilizado foi blocos casualizados com quatro repetições, em esquema fatorial $2 \times 3$, sendo duas doses de metribuzin $\left(0\right.$ e $\left.432 \mathrm{~g} \mathrm{ha}^{-1}\right)$ e três doses de $\mathrm{N}\left(0,30\right.$ e $\left.90 \mathrm{~kg} \mathrm{ha}^{-1}\right)$. O metribuzin (Sencor 480 $\mathrm{SC}, 480 \mathrm{~L}^{-1} \mathrm{~g}$ i.a., SC, Bayer) foi aplicado no estádio de três folhas verdadeiras completamente expandidas. A aplicação for realizada com pulverizador costal pressurizado com $\mathrm{CO}_{2}$, equipado com pontas tipo leque 110.02 e calibrado para aspergir $200 \mathrm{~L} \mathrm{ha}^{-1}$ de calda. $\mathrm{O} \mathrm{N}$ foi distribuído a lanço, aos sete dias após aplicação do herbicida, com a utilização do nitrato de amônio.

As unidades experimentais dos três experimentos foram compostas por 5,5 metros de comprimento, com área da parcela de $10 \mathrm{~m}^{2}$. No entanto, a largura das parcelas se distinguiu de acordo com o local onde o experimento foi realizado. Assim, foi composto de cinco e quatro fileiras duplas de cenoura, nos experimentos dos locais A e B, respectivamente, e três fileiras triplas no experimento do local C. Em todos eles, foram desconsiderados meio metro de bordadura nas extremidades.

Todos experimentos receberam adubações de base e cobertura conforme exigência nutricional da mesma e características químicas das áreas (Tabela 1). Foram aplicados em semeadura 40, 600 e $120 \mathrm{~kg} \mathrm{ha}^{-1}$ de $\mathrm{N}, \mathrm{P}_{2} \mathrm{O}_{5}$ e $\mathrm{K}_{2} \mathrm{O}$, respectivamente, no local $\mathrm{A} ; 75,1120$ e 0 no local $\mathrm{B}$; e 40, 780 e 120 no local C. Ainda, a adubação de cobertura foi de 20, 200 e $280 \mathrm{~kg} \mathrm{ha}^{-1}$ de $\mathrm{N}, \mathrm{P}_{2} \mathrm{O}_{5}$ e $\mathrm{K}_{2} \mathrm{O}$ no local $\mathrm{A}$; 40, 460 e 760 no local B; e 40, 20 e 220 no local C. O manejo fitossanitário de pragas e doenças das áreas foi o comumente utilizado pelas fazendas, no qual as tomadas de decisão para o controle foram baseadas nos dados de monitoramento das mesmas. Não foi realizada nenhuma aplicação de herbicida, além do tratamento proposto. Todas as unidades experimentais foram mantidas no limpo, isto é, sem interferência de plantas daninhas, durante todo o período de execução do experimento, por meio de capinas manuais, sempre que necessário. 
(A)

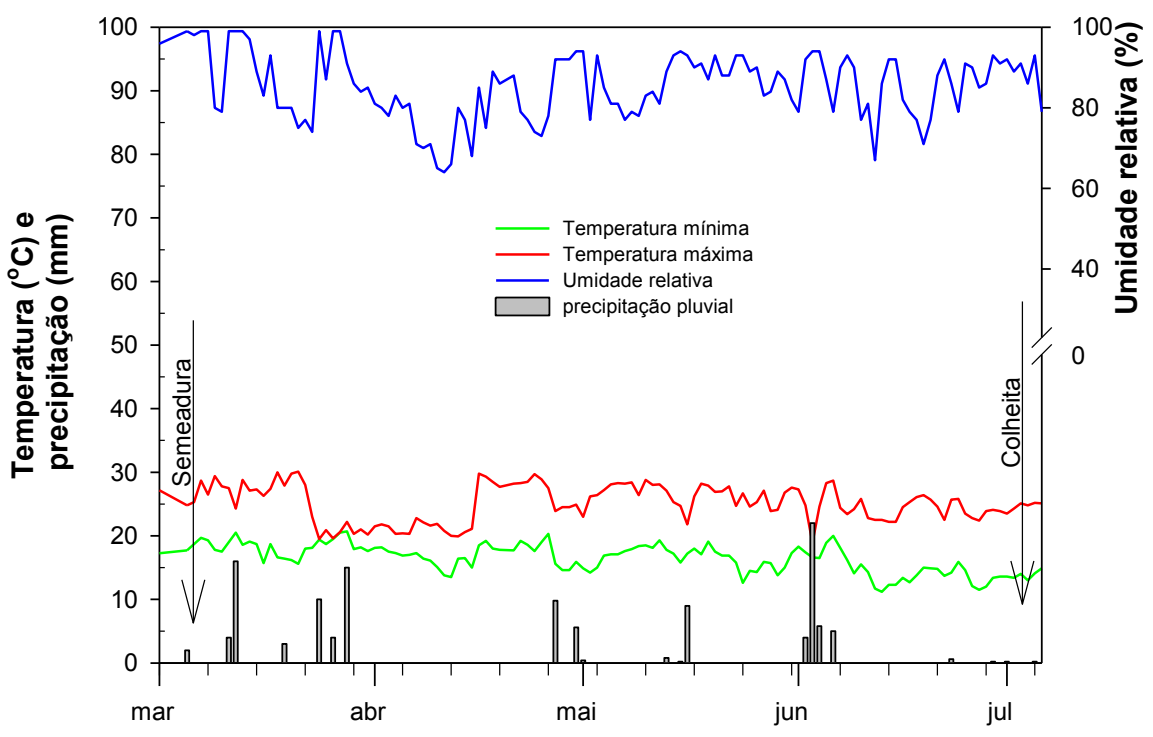

(B)

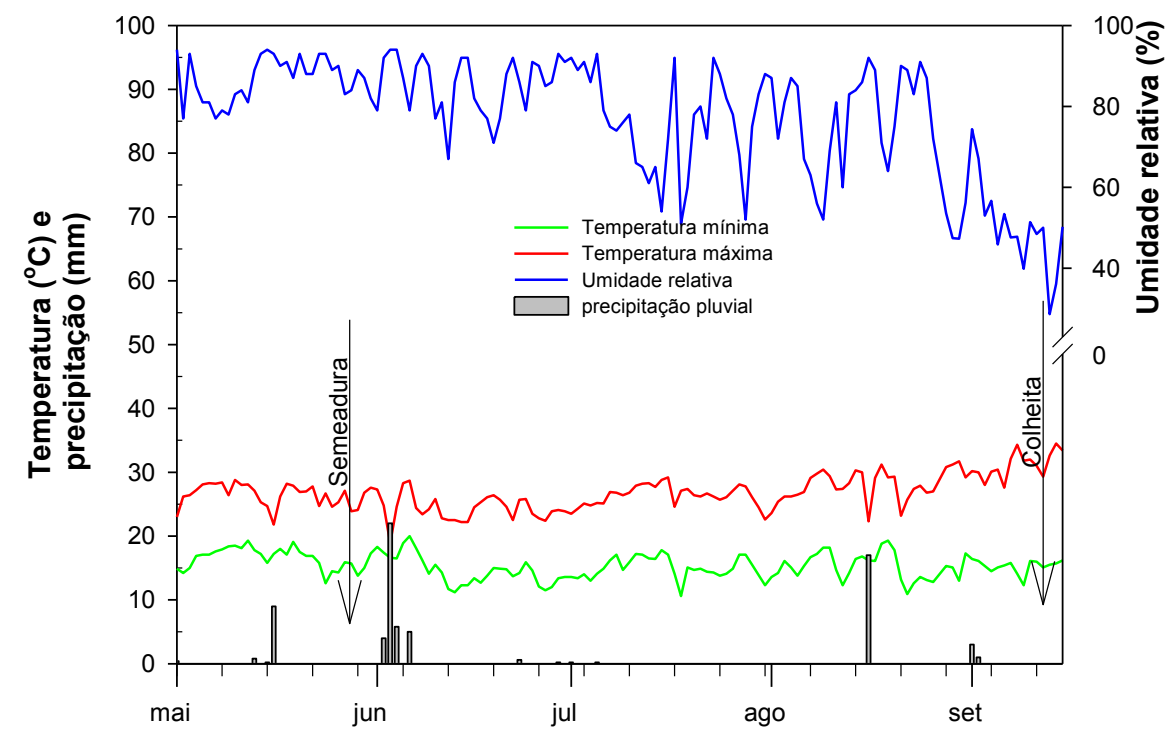

(C)

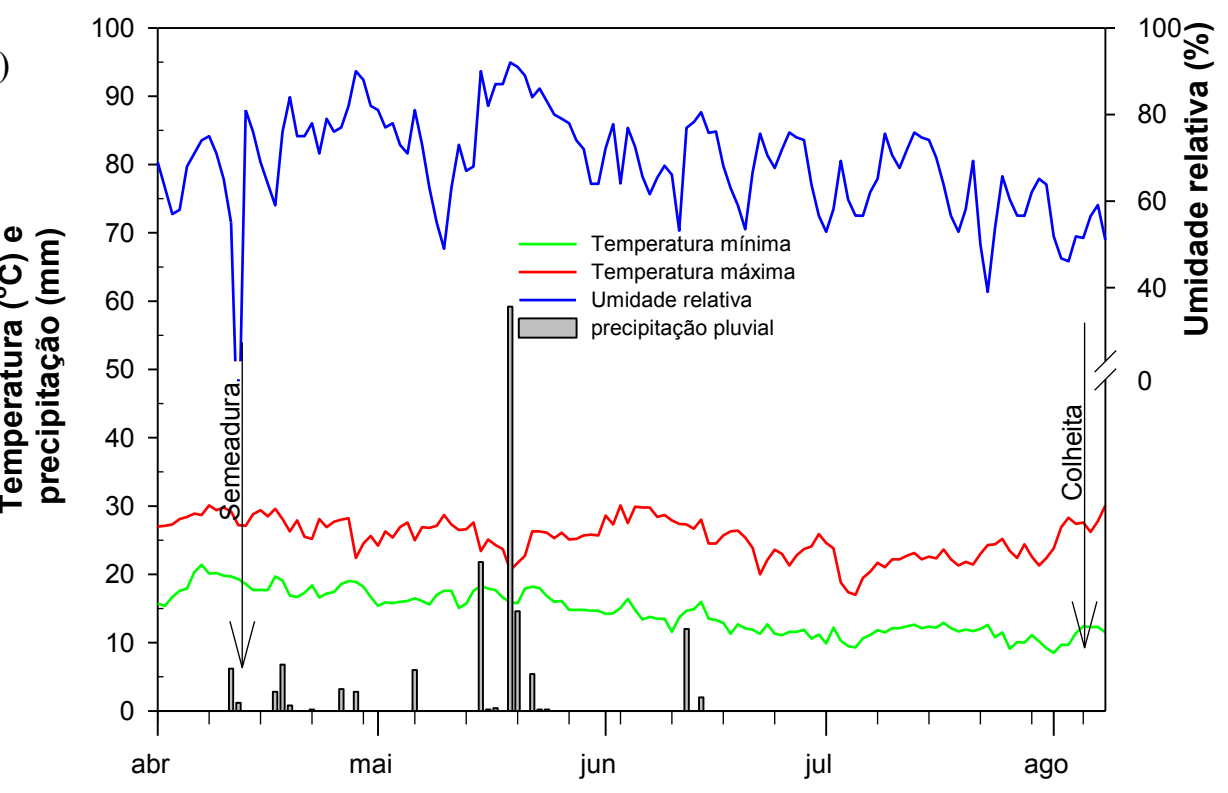

Figura 1. Temperatura mínima e máxima $\left({ }^{\circ} \mathrm{C}\right)$, umidade relativa do ar $(\%)$ e precipitação pluvial (mm) no local A (A), local B (B) e no local C (C). 
Aos 14 e 28 dias após a aplicação do nitrogênio (DAA), foram realizadas avaliações de comprimento e diâmetro de raízes, e massa da matéria seca de parte aérea (MSPA) e raiz das plantas (MSR). A avaliação for realizada em 20 plantas por parcela, coletadas aleatoriamente. A determinação do diâmetro foi realizada com paquímetro digital localizado a três centímetros abaixo da inserção do caule. Após as medições de crescimento, as plantas foram levadas para secagem em estufa de ventilação forçada de ar, a $70^{\circ} \mathrm{C}$, até atingir massa constante para determinação da massa da matéria seca.

Tabela 1. Caracterização química do solo das áreas experimentais, de 0-20 cm.

\begin{tabular}{cccccccc}
\hline \multirow{2}{*}{ Local } & $\begin{array}{c}\mathrm{pH} \\
\left(\mathrm{H}_{2} \mathrm{O}\right)\end{array}$ & $\begin{array}{c}\text { P- disponível } \\
(\mathrm{M}-1)\end{array}$ & $\mathrm{P}-\mathrm{rem}$ & $\mathrm{V}$ & $\mathrm{t}$ & M.O. & C.O. \\
\cline { 2 - 8 } & & $\mathrm{mg} \mathrm{dm}^{-3}$ & $\mathrm{mg} \mathrm{L}^{-1}$ & $\%$ & $\mathrm{cmol}_{\mathrm{c}} \mathrm{dm}^{-3}$ & $\mathrm{~g} \mathrm{dm}^{-3}$ \\
\hline $\mathrm{A}$ & 5,9 & 82,5 & 6,9 & 62,1 & 4,76 & 26,0 & 15,1 \\
$\mathrm{~B}$ & 5,8 & 22,3 & 9,6 & 51,7 & 4,60 & 24,0 & 13,9 \\
$\mathrm{C}$ & 6,8 & 158,4 & 24,3 & 56,3 & 5,02 & 22,0 & 12,8 \\
\hline
\end{tabular}

M-1 = Mehlich-1; P-rem = Fósforo remanescente; V = Saturação por bases; $\mathrm{t}$ = CTC efetiva; M.O = Matéria orgânica do solo; C.O = Carbono orgânico do solo.

Aos 120 DAA, foram coletadas 100 plantas por unidade experimental, na área útil, para determinação da produtividade comercial das raízes, entre 10 e $26 \mathrm{~cm}$ de comprimento, e não comercial, com raízes inferiores a 10 e superiores a $26 \mathrm{~cm}$ de comprimento e com presença de defeitos (CEAGESP, 2015).

Os dados foram submetidos a testes de homogeneidade das variâncias e normalidade dos resíduos (Shapiro Wilk?). Em seguida, os dados foram submetidos à análise de variância $(\mathrm{p}<0,05) \mathrm{e}$, as médias comparadas entre si pelo teste de Tukey $(\mathrm{p}<0,05)$.

\section{Resultados e Discussão}

Não houve interação entre o metribuzin e o nitrogênio nas variáveis estudadas, com exceção do diâmetro de raiz no local C, aos 14 DAA. Nesta, não houve diferença entre as doses de $\mathrm{N}$ na ausência do metribuzin. No entanto, a aplicação de $432 \mathrm{~g} \mathrm{ha}^{-1}$ de metribuzin e maior dose de $\mathrm{N}$ aumentou o diâmetro de raízes comparada às doses de 0 e $30 \mathrm{~kg} \mathrm{ha}^{-1}$ de $\mathrm{N}$ (Tabela 2). O diâmetro de raízes foi reduzido pela aplicação do metribuzin em todos os locais de cultivo. De forma geral, não houve diferença significativa entre as doses de $\mathrm{N}$ sobre esta variável. Resultado similar foi encontrado por Agyei e Bayor (2017), que não verificaram diferenças entre o tratamento controle, 0 de $\mathrm{N}$, e $34 \mathrm{~kg} \mathrm{ha}^{-1}$ de $\mathrm{N}$ sobre o diâmetro de raiz da cenoura. Murthy et al. (2016) e Moniruzzaman et al. (2013) verificaram correlação positiva entre o diâmetro de raízes e o aumento da fertilização do nitrogênio para doses até 75 e $100 \mathrm{~kg} \mathrm{ha}^{-1} \mathrm{de} \mathrm{N}$, respectivamente.

Tabela 2. Diâmetro de raiz (cm) em função de doses de metribuzin e N na cultura da cenoura aos 14 e 28 dias após aplicação (DAA) do nitrogênio.

\begin{tabular}{|c|c|c|c|c|c|c|c|c|}
\hline \multicolumn{9}{|c|}{ Diâmetro de raiz $(\mathrm{cm})$} \\
\hline & & & $14 \mathrm{DAA}^{/ 1}$ & & & $28 \mathrm{DA}$ & & \\
\hline \multirow{2}{*}{ Dose de metribuzin $\left(\mathrm{g} \mathrm{ha}^{-1}\right)$} & \multicolumn{3}{|c|}{ Dose de N $\left(\mathrm{kg} \mathrm{ha}^{-1}\right)$} & \multirow{2}{*}{ Média } & \multicolumn{3}{|c|}{ Dose de $\mathrm{N}\left(\mathrm{kg} \mathrm{ha}^{-1}\right)$} & \multirow{2}{*}{ Média } \\
\hline & 0 & 30 & 90 & & 0 & 30 & 90 & \\
\hline \multicolumn{9}{|c|}{ Local A } \\
\hline 0 & $1,5^{2}$ & 1,5 & 1,5 & $1,5 \mathrm{~A}$ & 2,4 & 2,5 & 2,6 & 2,5 \\
\hline 432 & 1,5 & 1,5 & 1,4 & $1,4 \mathrm{~B}$ & 2,4 & 2,5 & 2,5 & 2,5 \\
\hline Média & 1,5 & 1,5 & 1,5 & & $2,4 \mathrm{~b}$ & $2,5 \mathrm{ab}$ & $2,6 \mathrm{a}$ & \\
\hline $\mathrm{F}_{\mathrm{M}}=5,31^{*}$ & $\mathrm{~F}_{\mathrm{N}}=0,18^{\mathrm{ns}}$ & $\mathrm{F}_{\mathrm{i}}=1,09^{\mathrm{ns}}$ & C.V. $=5,1$ & $\mathrm{~F}_{\mathrm{M}}=0,45^{\mathrm{ns}}$ & $\mathrm{F}_{\mathrm{N}}=6,7^{* *}$ & $\mathrm{~F}_{\mathrm{I}}=0,56^{\mathrm{ns}}$ & & \\
\hline \multicolumn{9}{|c|}{ Local B } \\
\hline 0 & 1,2 & 1,2 & 1,1 & $1,2 \mathrm{~A}$ & 2,2 & 2,2 & 2,2 & $2,2 \mathrm{~A}$ \\
\hline 432 & 1,1 & 1,1 & 1,1 & $1,1 \mathrm{~B}$ & 2,0 & 2,1 & 2,1 & $2,1 \mathrm{~B}$ \\
\hline Média & 1,1 & 1,2 & 1,1 & & 2,1 & 2,1 & 2,1 & \\
\hline $\mathrm{F}_{\mathrm{M}}=5,84^{*}$ & $\mathrm{~F}_{\mathrm{N}}=0,42^{\mathrm{ns}}$ & $\mathrm{F}_{\mathrm{I}}=0,69^{\mathrm{ns}}$ & C.V. $=9,2$ & $\mathrm{~F}_{\mathrm{M}}=12,8^{* *}$ & $\mathrm{~F}_{\mathrm{N}}=0,08^{\mathrm{ns}}$ & $\mathrm{F}_{\mathrm{I}}=0,17^{\mathrm{ns}}$ & C. & \\
\hline \multicolumn{9}{|c|}{ Local C } \\
\hline 0 & $1,9 \mathrm{Aa}$ & $2,0 \mathrm{Aa}$ & $2,0 \mathrm{Aa}$ & 2,0 & 2,7 & 2,8 & 2,8 & $2,8 \mathrm{~A}$ \\
\hline 432 & $1,7 \mathrm{Bb}$ & $1,7 \mathrm{Bb}$ & $1,9 \mathrm{Aa}$ & 1,8 & 2,6 & 2,6 & 2,7 & $2,6 \mathrm{~B}$ \\
\hline Média & 1,8 & 1,9 & 1,9 & & 2,7 & 2,7 & 2,8 & \\
\hline $\mathrm{F}_{\mathrm{M}}=39,2^{* *}$ & $\mathrm{~F}_{\mathrm{N}}=3,14^{\mathrm{ns}}$ & $\mathrm{F}_{\mathrm{I}}=4,00^{* *}$ & C.V. $=4,0$ & $\mathrm{~F}_{\mathrm{M}}=6,62^{*}$ & $\mathrm{~F}_{\mathrm{N}}=1,65^{\mathrm{ns}}$ & $\mathrm{F}_{\mathrm{I}}=0,21^{\mathrm{ns}}$ & & \\
\hline
\end{tabular}

${ }^{12}$ Médias seguidas pela mesma letra, minúscula na linha e maiúscula na coluna não diferem entre si pelo teste de Tukey e $\mathrm{F}$, respectivamente, a $5 \%$ de probabilidade. ${ }^{\text {ns }}$ não significativo $(\mathrm{p} \geq 0.05)$. * significativo ao nível $(\mathrm{p}<0.05)$. ${ }^{* *}$ significativo ao nível $(\mathrm{p}<0.01) . \mathrm{F}_{\mathrm{M}}: \mathrm{F}$ calculado para fator metribuzin. $\mathrm{F}_{\mathrm{N}}: \mathrm{F}$ calculado para fator nitrogênio. $\mathrm{F}_{\mathrm{I}}$ : $\mathrm{F}$ calculado para interação metribuzin $\mathrm{x}$ nitrogênio. 
Não houve efeito do metribuzin e das doses de $\mathrm{N}$ sobre o comprimento de raízes da cenoura, exceto para o experimento realizado no local A aos 28 DAA. Na ausência de adubação nitrogenada em cobertura, o comprimento de raiz foi 3,6\% maior em relação a dose de $90 \mathrm{~kg} \mathrm{ha}^{-1}$ de $\mathrm{N}$ (Tabela 3). Agyei e Bayor (2017) não reportaram diferenças no comprimento de raiz após aplicação de fertilizante mineral na dose de $34 \mathrm{~kg} \mathrm{ha}^{-1}$ de $\mathrm{N}$, fertilizante orgânico a base de estrume de aves e ausência de N. As principais evidências envolvem que ambientes enriquecidos com $\mathrm{N}$ podem proporcionar à planta consumo excessivo do nutriente com alto custo energético para aumentar o diâmetro e o comprimento da raiz. Entretanto, Moniruzzaman et al. (2013) verificaram que a aplicação de 70 e $130 \mathrm{~kg} \mathrm{ha}^{-1}$ de $\mathrm{N}$ não diferiram entre si, com aumento do comprimento da raiz da cenoura comparado a ausência de fertilização com $\mathrm{N}$ na cultura.

Tabela 3. Comprimento de raiz $(\mathrm{cm})$ em função de doses de metribuzin e $\mathrm{N}$ na cultura da cenoura aos 14 e 28 dias após aplicação do nitrogênio.

\begin{tabular}{|c|c|c|c|c|c|c|c|c|}
\hline \multicolumn{9}{|c|}{ Comprimento de raiz $(\mathrm{cm})$} \\
\hline & & $14 \mathrm{DAA}^{11}$ & & & & DAA & & \\
\hline \multirow{2}{*}{ Dose de metribuzin $\left(\mathrm{g} \mathrm{ha}^{-1}\right)$} & \multicolumn{3}{|c|}{ Dose de $N\left(\mathrm{~kg} \mathrm{ha}^{-1}\right)$} & \multirow{2}{*}{ Média } & \multicolumn{3}{|c|}{ Dose de N $\left(\mathrm{kg} \mathrm{ha}^{-1}\right)$} & \multirow{2}{*}{ Média } \\
\hline & 0 & 30 & 90 & & 0 & 30 & 90 & \\
\hline \multicolumn{9}{|c|}{ Local A } \\
\hline 0 & $21,1^{/ 2}$ & 20,8 & 21,2 & 21,0 & 22,0 & 21,2 & 21,2 & 21,5 \\
\hline 432 & 21,3 & 21,2 & 20,8 & 21,1 & 21,9 & 21,6 & 21,1 & 21,5 \\
\hline Média & 21,2 & 21,0 & 21,0 & & $22,0 \mathrm{a}$ & $21,4 \mathrm{ab}$ & $21,2 \mathrm{~b}$ & \\
\hline $\mathrm{F}_{\mathrm{M}}=0,15^{\mathrm{ns}}$ & $\mathrm{F}_{\mathrm{N}}=0,60^{\mathrm{ns}}$ & $\mathrm{F}_{\mathrm{I}}=1,64^{\mathrm{ns}}$ & C.V. $=2,0$ & $\mathrm{~F}_{\mathrm{M}}=0,04^{\mathrm{ns}}$ & $\mathrm{F}_{\mathrm{N}}=5,74^{*}$ & $\mathrm{~F}_{\mathrm{I}}=0,75^{\mathrm{ns}}$ & & $=2,3$ \\
\hline \multicolumn{9}{|c|}{ Local B } \\
\hline 0 & 18,9 & 19,4 & 18,4 & 18,9 & 20,4 & 21,1 & 20,9 & 20,8 \\
\hline 432 & 18,3 & 18,2 & 18,8 & 18,4 & 20,0 & 20,1 & 20,6 & 20,2 \\
\hline Média & 18,6 & 18,8 & 18,6 & & 20,2 & 20,6 & 20,7 & \\
\hline $\mathrm{F}_{\mathrm{M}}=2,0^{\mathrm{ns}}$ & $\mathrm{F}_{\mathrm{N}}=0,12^{\mathrm{ns}}$ & $\mathrm{F}_{\mathrm{I}}=1,83^{\mathrm{ns}}$ & C.V. $=4,7$ & $\mathrm{~F}_{\mathrm{M}}=3,32^{\mathrm{ns}}$ & $\mathrm{F}_{\mathrm{N}}=0,9^{\mathrm{ns}}$ & $\mathrm{F}_{\mathrm{I}}=0,52^{\mathrm{ns}}$ & & $=3,8$ \\
\hline \multicolumn{9}{|c|}{ Local C } \\
\hline 0 & 20,6 & 20,8 & 20,8 & 20,8 & 24,2 & 22,3 & 22,5 & 23,0 \\
\hline 432 & 20,8 & 21,1 & 19,9 & 20,6 & 22,5 & 22,8 & 21,5 & 22,3 \\
\hline Média & 20,7 & 21,0 & 20,3 & & 23,3 & 22,5 & 22,0 & \\
\hline $\mathrm{F}_{\mathrm{M}}=012^{\mathrm{ns}}$ & $\mathrm{F}_{\mathrm{N}}=0,8^{\mathrm{ns}}$ & $\mathrm{F}_{\mathrm{I}}=0,77^{\mathrm{ns}}$ & C.V. $=4,8$ & $\mathrm{~F}_{\mathrm{M}}=2,95^{\mathrm{ns}}$ & $\mathrm{F}_{\mathrm{N}}=3,52^{\mathrm{ns}}$ & $\mathrm{F}_{\mathrm{I}}=2,28^{\mathrm{ns}}$ & \multicolumn{2}{|c|}{ C.V. $=4,5$} \\
\hline
\end{tabular}

${ }^{/ 1} \mathrm{DAA}=$ Dias após aplicação de doses de nitrogênio. ${ }^{/ 2}$ Médias seguidas pela mesma letra, minúscula na linha, não diferem entre si pelo teste de Tukey e F, respectivamente, a $5 \%$ de probabilidade. ${ }^{\text {ns }}$ não significativo (p $\geq 0.05$ ). ${ }^{*}$ significativo ao nível de $5 \%$ de probabilidade $(0.01 \leq \mathrm{p}<0.05) . \mathrm{F}_{\mathrm{M}}: \mathrm{F}$ calculado para fator metribuzin. $\mathrm{F}_{\mathrm{N}}: \mathrm{F}$ calculado para fator nitrogênio. $\mathrm{F}_{\mathrm{I}}: \mathrm{F}$ calculado para interação metribuzin $\mathrm{x}$ nitrogênio

A divergência entre os trabalhos sobre a influência do nitrogênio no comprimento e diâmetro da raiz da cenoura, pode ser explicada pelos diferentes ambientes de cultivo e produtividades alcançadas. Desta forma, a realização deste trabalho em solo $\mathrm{cm}$ menor matéria orgânica poderia proporcionar resposta positiva a aplicação deste nutriente. No entanto, a cultura da cenoura apresenta sistema radicular pouco agressivo e baixa eficiência de mobilização dos nutrientes, com alta exigência de $\mathrm{N}$ para obtenção de altas produtividades.

Não foram verificados sintomas visuais de intoxicação na cultura da cenoura. Somente no experimento realizado no Local $\mathrm{C}$, aos 14 DAA, o metribuzin reduziu a massa da matéria seca da parte aérea da cenoura (Tabela 4). Em todos os outros, não houve influência do herbicida e do
$\mathrm{N}$ sobre esta variável. Este resultado evidencia que a dose de $432 \mathrm{~g} \mathrm{ha}^{-1}$ de metribuzin não foi suficiente para reduzir a massa da parte aérea das plantas, demonstrando que a cultura é tolerante ao metribuzin para a dose avaliada. Carneiro et al. (2017) evidenciaram que 432 g i.a. ha ${ }^{-1}$ do herbicida metribuzin foi a maior dose seletiva à cultura, sem afetar negativamente a produtividade de raízes. No entanto, Carneiro (2016) verificou que a dose de $576 \mathrm{~g} \mathrm{ha}^{-1} \mathrm{de}$ metribuzin reduziu a MSPA, com redução da produtividade comercial da cultura. Para o manejo do N, a aplicação de $90 \mathrm{~kg} \mathrm{ha}^{-1}$ de $\mathrm{N}$ não propiciou incrementos na MSPA, diferindo dos resultados encontrados por Murthy et al. (2016) que verificou aumento da massa seca da parte aérea para doses de até $75 \mathrm{~kg} \mathrm{ha}^{-1}$ de $\mathrm{N}$. 
F. O. XAVIER

Tabela 4. Massa da matéria seca da parte aérea (g/planta) em função de doses de metribuzin e N na cultura da cenoura aos 14 e 28 dias após aplicação do nitrogênio.

\begin{tabular}{|c|c|c|c|c|c|c|c|c|}
\hline \multicolumn{9}{|c|}{ Massa da matéria seca da parte aérea ( $\mathrm{g} /$ planta) } \\
\hline \multicolumn{4}{|c|}{$14 \mathrm{DAA}^{/ 1}$} & \multicolumn{5}{|c|}{28 DAA } \\
\hline \multirow{2}{*}{ Dose de metribuzin $\left(\mathrm{g} \mathrm{ha}^{-1}\right)$} & \multicolumn{3}{|c|}{ Dose de $\mathrm{N}\left(\mathrm{kg} \mathrm{ha}^{-1}\right)$} & \multirow{2}{*}{ Média } & \multicolumn{3}{|c|}{ Dose de $\mathrm{N}\left(\mathrm{kg} \mathrm{ha}^{-1}\right)$} & \multirow[t]{2}{*}{ Médic } \\
\hline & 0 & 30 & 90 & & 0 & 30 & 90 & \\
\hline \multicolumn{9}{|c|}{ Local A } \\
\hline 0 & $2,6^{2}$ & 2,5 & 2,5 & 2,5 & 3,8 & 4,0 & 4,5 & 4,1 \\
\hline 432 & 2,5 & 2,4 & 2,5 & 2,4 & 4,0 & 4,3 & 4,1 & 4,1 \\
\hline Média & 2,5 & 2,5 & 2,5 & & 3,9 & 4,1 & 4,3 & \\
\hline $\mathrm{F}_{\mathrm{M}}=1,51^{\mathrm{ns}}$ & $\mathrm{F}_{\mathrm{N}}=0,19^{\mathrm{ns}}$ & $\mathrm{F}_{\mathrm{I}}=0,02^{\mathrm{ns}}$ & C. $V=8,9$ & $\mathrm{~F}_{\mathrm{M}}=0,06^{\mathrm{ns}}$ & $\mathrm{F}_{\mathrm{N}}=1,23^{\mathrm{ns}}$ & $\mathrm{F}_{\mathrm{I}}=1,03^{\mathrm{ns}}$ & & C.V. $=12,1$ \\
\hline \multicolumn{9}{|c|}{ Local B } \\
\hline 0 & 2,1 & 2,2 & 1,9 & 2,1 & $-{ }^{3}$ & - & - & - \\
\hline 432 & 2,0 & 1,9 & 2,0 & 2,0 & - & - & - & - \\
\hline Média & 2,0 & 2,1 & 1,9 & & - & - & - & \\
\hline $\mathrm{F}_{\mathrm{M}}=1,57^{\mathrm{ns}}$ & $\mathrm{F}_{\mathrm{N}}=0,61^{\mathrm{ns}}$ & $\mathrm{F}_{\mathrm{I}}=0,91^{\mathrm{ns}}$ & C.V. $=12,5$ & - & - & - & & - \\
\hline \multicolumn{9}{|c|}{ Local C } \\
\hline 0 & 3,1 & 3,0 & 2,9 & $3,0 \mathrm{~A}$ & 4,4 & 4,6 & 4,7 & 4,6 \\
\hline 432 & 2,9 & 2,7 & 2,7 & $2,7 \mathrm{~B}$ & 4,3 & 3,9 & 4,2 & 4,1 \\
\hline Média & 3,0 & 2,8 & 2,8 & & 4,3 & 4,3 & 4,5 & \\
\hline $\mathrm{F}_{\mathrm{M}}=6,15^{*}$ & $\mathrm{~F}_{\mathrm{N}}=1,42^{\mathrm{ns}}$ & $\mathrm{F}_{\mathrm{I}}=0,08^{\mathrm{ns}}$ & C.V. $=8,6$ & $\mathrm{~F}_{\mathrm{M}}=4,46^{\mathrm{ns}}$ & $\mathrm{F}_{\mathrm{N}}=0,28^{\mathrm{ns}}$ & $\mathrm{F}_{\mathrm{I}}=0,54^{\mathrm{ns}}$ & & C.V. $=12,6$ \\
\hline
\end{tabular}

${ }^{/ 1} \mathrm{DAA}=$ Dias após aplicação de doses de nitrogênio. ${ }^{/ 2}$ Médias seguidas pela mesma letra, maiúscula na coluna, não diferem entre si pelo teste de Tukey e $\mathrm{F}$, respectivamente, a $5 \%$ de probabilidade. ${ }^{\text {ns }}$ não significativo (p $\left.\geq 0.05\right) .{ }^{*}$ significativo ao nível de $5 \%$ de probabilidade $(0.01 \leq p<0.05)$. $F_{M}: F$ calculado para fator metribuzin. $F_{N}: F$ calculado para fator nitrogênio. $F_{I}: F$ calculado para interação metribuzin $x$ nitrogênio. ${ }^{/ 3}$ Dados perdidos.

A MSR foi reduzida pelo metribuzin nas duas épocas de avaliação, nos locais B e C (Tabela 5). No local A, aos 14 DAT, não houve influência do herbicida sobre a MSR, enquanto aos 28 DAA foi observado maior MSR na presença do metribuzin. O N não influenciou a MSR em nenhum dos experimentos realizados. Murthy et al. (2016) verificaram incremento na massa da matéria seca da raiz da cenoura com o aumento das doses de N. No entanto, a redução na MSR causada pelo metribuzin no presente trabalho não segue a mesma tendência da produtividade de raízes (Tabela 6). Desta forma, pode-se dizer que a cultura foi capaz de se recuperar entre esta avaliação e o período de colheita.

Tabela 5. Massa da matéria seca da raiz (g/planta) em função de doses de metribuzin e $\mathrm{N}$ na cultura da cenoura aos 14 e 28 dias após aplicação do nitrogênio.

\begin{tabular}{|c|c|c|c|c|c|c|c|c|}
\hline \multicolumn{9}{|c|}{ Massa da matéria seca da raiz (g/planta) } \\
\hline & \multicolumn{3}{|c|}{$14 \mathrm{DAA}^{11}$} & \multicolumn{4}{|c|}{28 DAA } & \multirow{3}{*}{ Média } \\
\hline \multirow{2}{*}{ Dose de metribuzin $\left(\mathrm{g} \mathrm{ha}^{-1}\right)$} & \multicolumn{3}{|c|}{ Dose de N $\left(\mathrm{kg} \mathrm{ha}^{-1}\right)$} & \multirow{2}{*}{ Média } & \multicolumn{3}{|c|}{ Dose de $\mathrm{N}\left(\mathrm{kg} \mathrm{ha}^{-1}\right)$} & \\
\hline & 0 & 30 & 90 & & 0 & 30 & 90 & \\
\hline \multicolumn{9}{|c|}{ Local A } \\
\hline 0 & $1,4^{12}$ & 1,3 & 1,5 & 1,4 & 4,5 & 4,6 & 5,0 & $4,7 \mathrm{~B}$ \\
\hline 432 & 1,3 & 1,3 & 1,3 & 1,3 & 5,2 & 5,2 & 4,9 & $5,1 \mathrm{~A}$ \\
\hline Média & 1,4 & 1,3 & 1,4 & & 4,8 & 4,9 & 5,0 & \\
\hline $\mathrm{F}_{\mathrm{M}}=1,07^{\mathrm{ns}}$ & $\mathrm{F}_{\mathrm{N}}=0,99^{\mathrm{ns}}$ & $\mathrm{F}_{\mathrm{I}}=0,21^{\mathrm{ns}}$ & & $=14,5$ & $\mathrm{~F}_{\mathrm{M}}=5,27^{*}$ & $\mathrm{~F}_{\mathrm{N}}=0,27^{\mathrm{ns}}$ & $\mathrm{F}_{\mathrm{I}}=2,46^{\mathrm{ns}}$ & C.V. $=8,2$ \\
\hline \multicolumn{9}{|c|}{ Local B } \\
\hline 0 & 1,1 & 1,1 & 1,0 & $1,1 \mathrm{~A}$ & 3,5 & 3,6 & 3,1 & $3,4 \mathrm{~A}$ \\
\hline 432 & 0,9 & 0,9 & 0,9 & $0,9 \mathrm{~B}$ & 3,2 & 2,9 & 2,7 & $3,0 \mathrm{~B}$ \\
\hline Média & 1,0 & 1,0 & 1,0 & & 3,3 & 3,3 & 2,9 & \\
\hline $\mathrm{F}_{\mathrm{M}}=5,24^{*}$ & $\mathrm{~F}_{\mathrm{N}}=0,21^{\mathrm{ns}}$ & $\mathrm{F}_{\mathrm{I}}=0,38^{\mathrm{ns}}$ & & $=16,9$ & $\mathrm{~F}_{\mathrm{M}}=5,40^{*}$ & $\mathrm{~F}_{\mathrm{N}}=2,10^{\mathrm{ns}}$ & $\mathrm{F}_{\mathrm{I}}=0,52^{\mathrm{ns}}$ & C.V. $=14,5$ \\
\hline \multicolumn{9}{|c|}{ Local C } \\
\hline 0 & 2,3 & 2,3 & 2,2 & $2,3 \mathrm{~A}$ & 6,0 & 6,2 & 6,1 & $6,1 \mathrm{~A}$ \\
\hline 432 & 1,9 & 1,7 & 2,0 & $1,9 \mathrm{~B}$ & 5,6 & 5,3 & 5,4 & $5,4 \mathrm{~B}$ \\
\hline Média & 2,1 & 2,0 & 2,1 & & 5,8 & 5,8 & 5,7 & \\
\hline $\mathrm{F}_{\mathrm{M}}=47,0^{* *}$ & $\mathrm{~F}_{\mathrm{N}}=0,88^{\mathrm{ns}}$ & $\mathrm{F}_{\mathrm{I}}=3,08^{\mathrm{ns}}$ & & $=6,9$ & $\mathrm{~F}_{\mathrm{M}}=9,1^{* *}$ & $\mathrm{~F}_{\mathrm{N}}=0,02^{\mathrm{ns}}$ & $\mathrm{F}_{\mathrm{I}}=0,44^{\mathrm{ns}}$ & C.V. $=9,8$ \\
\hline
\end{tabular}

${ }^{/ 1} \mathrm{DAA}=$ Dias após aplicação de doses de nitrogênio. ${ }^{2}$ Médias seguidas pela mesma letra, maiúscula na coluna, não diferem entre si pelo teste de Tukey e F, respectivamente, a $5 \%$ de probabilidade. ${ }^{\text {ns }}$ não significativo $(\mathrm{p} \geq 0.05)$. ${ }^{*}$ significativo ao nível de $5 \%$ de probabilidade $(0.01 \leq \mathrm{p}<0.05)$. ${ }^{* *}$ significativo ao nível de $1 \%$ de probabilidade $(\mathrm{p}<0.01)$. $\mathrm{F}_{\mathrm{M}}$ : $\mathrm{F}$ calculado para fator metribuzin. $\mathrm{F}_{\mathrm{N}}: \mathrm{F}$ calculado para fator nitrogênio. $\mathrm{F}_{\mathrm{I}}: \mathrm{F}$ calculado para interação metribuzin $\mathrm{x}$ nitrogênio. 
Para produtividade comercial e total não foram influenciadas pelo metribuzin em nenhum dos experimentos realizados. Ainda, nos locais $\mathrm{A}$ e $\mathrm{B}$, não houve influência do $\mathrm{N}$ sobre estas variáveis (Tabela 6). No entanto, no local C, a maior dose $\left(90 \mathrm{~kg} \mathrm{ha}{ }^{-1}\right)$ propiciou um aumento de aproximadamente $15 \%$ na produtividade comercial da cenoura em relação às duas menores doses. Estes resultados são diferentes dos encontrados por Carneiro et al. (2017), que verificaram redução da produtividade total da cenoura na dose de $432 \mathrm{~g} \mathrm{ha}^{-1}$ de metribuzin, comparado ao controle em cultivo no inverno. Silva et al. (2017) relataram redução da produtividade comercial da cenoura, cultivar de inverno, com a aplicação de $288 \mathrm{~g} \mathrm{ha}^{-1}$ de metribuzin. Repostas diferentes na produtividade comercial de cenoura em ambientes diferentes também foram observadas por Pereira et al. (2016) e Pereira et al. (2015), na qual os autores afirmam que esta variação ocorreu pelas características distintas de solo onde as plantas de cenoura foram cultivadas.

Tabela 6. Produtividade comercial e total $\left(\mathrm{t} \mathrm{ha}^{-1}\right)$ em função das doses de metribuzin e $\mathrm{N}$ na cultura da cenoura. Rio Paranaíba, MG (2018).

\begin{tabular}{|c|c|c|c|c|}
\hline \multicolumn{5}{|c|}{ Produtividade Comercial $\left(\mathrm{t} \mathrm{ha}^{-1}\right)$} \\
\hline \multirow{2}{*}{ Doses de metribuzin $\left(\mathrm{g} \mathrm{ha}^{-1}\right)$} & \multicolumn{3}{|c|}{ Doses de $\mathrm{N}\left(\mathrm{kg} \mathrm{ha}^{-1}\right)$} & \multirow{2}{*}{ Média } \\
\hline & 0 & 30 & 90 & \\
\hline \multicolumn{5}{|c|}{ Local A } \\
\hline 0 & 49,7 & 51,0 & 46,3 & 49,0 \\
\hline 432 & 47,7 & 49,9 & 46,2 & 47,9 \\
\hline Média & 48,7 & 50,4 & 46,3 & \multirow[b]{2}{*}{ C.V. $(\%)=9,2$} \\
\hline $\mathrm{F}_{\mathrm{Met}}=0,34^{\mathrm{ns}}$ & \multicolumn{2}{|c|}{$\mathrm{F}_{\mathrm{Nit}}=1,76^{\mathrm{ns}}$} & $\mathrm{F}_{\text {int }}=0,09^{\mathrm{ns}}$ & \\
\hline \multicolumn{5}{|c|}{ Local B } \\
\hline 0 & 52,3 & 54,5 & 56,1 & 54,3 \\
\hline 432 & 53,8 & 56,1 & 52,7 & 54,2 \\
\hline Média & 53,1 & 55,3 & 54,4 & \multirow[b]{2}{*}{ C.V. $(\%)=11,0$} \\
\hline $\mathrm{F}_{\mathrm{Met}}=0,00^{\text {ns }}$ & \multicolumn{2}{|c|}{$\mathrm{F}_{\mathrm{Nit}}=0,28^{\mathrm{ns}}$} & $\mathrm{F}_{\text {int }}=0,46^{\mathrm{ns}}$ & \\
\hline \multicolumn{5}{|c|}{ Local C } \\
\hline 0 & 62,1 & 61,6 & 72,4 & 65,4 \\
\hline 432 & 64,6 & 66,0 & 74,0 & 68,2 \\
\hline Média & $63,4 \mathrm{~b}$ & $63,8 \mathrm{~b}$ & $73,2 \mathrm{a}$ & \multirow[b]{2}{*}{ C.V. $(\%)=7,4$} \\
\hline $\mathrm{F}_{\mathrm{Met}}=1,98^{\mathrm{ns}}$ & \multicolumn{2}{|c|}{$\mathrm{F}_{\mathrm{Nit}}=10,02 * *$} & $\mathrm{~F}_{\text {int }}=0,16^{\mathrm{ns}}$ & \\
\hline \multicolumn{5}{|c|}{ Produtividade total $\left(\mathrm{t} \mathrm{ha}^{-1}\right)$} \\
\hline \multicolumn{5}{|c|}{ Local A } \\
\hline 0 & 55,6 & 57,2 & 53,5 & 55,4 \\
\hline 432 & 52,3 & 54,1 & 50,5 & 52,3 \\
\hline Média & 54,0 & 55,7 & 52,0 & \multirow[b]{2}{*}{ C.V. $(\%)=7,5$} \\
\hline $\mathrm{F}_{\mathrm{Met}}=3,64^{\mathrm{ns}}$ & \multicolumn{2}{|c|}{$\mathrm{F}_{\mathrm{Nit}}=1,67^{\mathrm{ns}}$} & $\mathrm{F}_{\mathrm{int}}=0,00^{\mathrm{ns}}$ & \\
\hline \multicolumn{5}{|c|}{ Local B } \\
\hline 0 & 59,1 & 57,8 & 59,1 & 58,7 \\
\hline 432 & 55,4 & 58,3 & 54,1 & 55,9 \\
\hline Média & 57,6 & 58,0 & 57,0 & \multirow[b]{2}{*}{ C.V. $(\%)=10,9$} \\
\hline $\mathrm{F}_{\mathrm{Met}}=1,16^{\mathrm{ns}}$ & \multicolumn{2}{|c|}{$\mathrm{F}_{\mathrm{Nit}}=0,11^{\mathrm{ns}}$} & $\mathrm{F}_{\text {int }}=0,43^{\mathrm{ns}}$ & \\
\hline \multicolumn{5}{|c|}{ Local C } \\
\hline 0 & 71,0 & 70,6 & 84,9 & 75,5 \\
\hline 432 & 77,7 & 73,2 & 84,6 & 78,5 \\
\hline Média & $74,3 \mathrm{~b}$ & $71,9 \mathrm{~b}$ & $84,8 \mathrm{a}$ & \\
\hline $\mathrm{F}_{\mathrm{Met}}=1,86^{\mathrm{ns}}$ & \multicolumn{2}{|c|}{$\mathrm{F}_{\mathrm{Nit}}=13,01 * *$} & $\mathrm{~F}_{\text {int }}=0,86^{\mathrm{ns}}$ & C.V. $(\%)=7,0$ \\
\hline
\end{tabular}

Médias seguidas pela mesma letra, minúscula na linha não diferem entre si pelo teste de Tukey e F, respectivamente, a $5 \%$ de probabilidade. ${ }^{\mathrm{ns}}$ não significativo $(\mathrm{p} \geq 0.05)$. ${ }^{* *}$ significativo ao nível de $1 \%$ de probabilidade $(\mathrm{p}<0.01) . \mathrm{F}_{\mathrm{M}}$ : $\mathrm{F}$ calculado para fator metribuzin. $\mathrm{F}_{\mathrm{N}}$ : $\mathrm{F}$ calculado para fator nitrogênio. $\mathrm{F}_{\mathrm{I}}$ : $\mathrm{F}$ calculado para interação metribuzin x nitrogênio.

De forma geral, o comprimento das raízes não foi alterado com a aplicação do metribuzin, que reduziu a MSR da cenoura devido redução do diâmetro. No entanto, esta redução afetou a produtividade da cultura. Os resultados evidenciam que o aumento da produtividade verificado no experimento conduzido no local $\mathrm{C}$ devido aplicação do $\mathrm{N}$, corrobora com os dados encontrados por Dezordi et al. (2015), na qual reportou que a cultura da cenoura apresenta resposta ao N. Murthy et al. (2016) e Moniruzzaman et al. (2013) verificaram incrementos no rendimento da cenoura em resposta à aplicação do nitrogênio de doses até $100 \mathrm{~kg} \mathrm{ha}^{-1}$.

$\mathrm{O}$ local $\mathrm{C}$ foi o que propiciou maiores valores de produtividade total e comercial de cenoura. Desta forma, pode-se inferir que para se alcançar alto rendimento para a cultura da cenoura é necessário maior demanda por nutrientes. Assim, este cultivo foi responsivo à maior dose de N aplicada. Portanto, cultivos de cenoura com produtividade elevada, podem responder a fertilizações nitrogenadas maiores às normalmente utilizadas pelos 
produtores de cenoura. No entanto, aumento na produtividade não foi influenciado pelo metribuzin, e não foi observada influência do herbicida na dinâmica deste nutriente na planta.

\section{Conclusões}

A aplicação do metribuzin associado à fertilização de $\mathrm{N}$ não influencia no crescimento axial ou radial da raiz da cenoura e, consequentemente, não altera a produtividade.

\section{Referências}

AGYEI, B. O.; BAYOR, H. Effect of poultry manure and nitrogen, phosphorus, and Potassium (15:15:15) Soil amendment on growth and yield of carrot (Daucus carota). International Journal of Biological, Biomolecular, Agricultural, Food and Biotechnological Engineering, v. 11, n. 2, p. 81-86, 2017.

CARNEIRO, G. D. O. P. Ácido salicílico e sacarose na redução de estresse causado pelo metribuzin na cultura da cenoura. 2016. 50 f. Tese (Mestrado em Agronomia) Universidade Federal de Viçosa, Rio Paranaíba, 2016.

CARNEIRO, G. D. O. P. et al. Selectivity of metribuzin in postemergence of culture of carrot. Planta Daninha, v. 35, p. 1-6, 2017.

CEAGESP. Companhia de entrepostos e armazéns gerais de São Paulo. Normas de classificação - cenoura. São Paulo. 2015. 5 p. (Programa para modernização da horticultura).

DEZORDI, L. R. et al. Nutrient demand of the carrot crop. African Journal of Agricultural Research, v. 10, n. 35, p. 3533-3542, 2015.

JENSEN, K. I. N.; DOOHAN, D. J.; SPECHT, E. G. Response of processing carrot to metribuzin on mineral soils in Nova Scotia. Canadian Jornal of Plant Science, v. 84, n. 2, p. 669-676, 2004.

MONIRUZZAMAN, M. et al. Effect of nitrogen on the growth and yield od carrot (Daucus carota L.). The Agriculturist, v. 11, n. 1, p. 76-81, 2013.

MURTHY, G. N. et al. Influence of different levels of nitrogen and phosphorous on growth and yield of carrot in high altitude and tribal zone of Andhra Pradesh. The Journal of Research Angrau, v. 44, n. 1/2, p. 67-73, 2016.

OLIVEIRA, D. M. Produtividade de cenoura em função de doses de nitrogênio e épocas de plantio. 2015. $26 \mathrm{f}$. Dissertação (Mestrado em Manejo de Solo e Água) Universidade Federal Rural do Semi-Árido, Mossoró - RN, 2015.
PEREIRA, G. A. M. et al. Crescimento de cultivares de cenoura em diferentes ambientes. Comunicata Scientiae, v. 6, n. 3, p. 317-325, 2016.

PEREIRA, G. A. M. et al. Performance of carrot genotypes at two Jequitinhonha valley sites. Semina: Ciências Agrárias, v. 36, n. 2, p. 4059-4069, 2015.

SILVA, G. S. et al. Use of metribuzin, associated with different foliar fertilizers, on carrot crops. Revista Colombiana de Ciências Hortícolas, v. 11, n. 2, p. 351358, 2017.

SILVA, A. A.; SILVA, J. F. Tópicos em manejo de plantas daninhas. Viçosa: Ed. UFV, 367 p. 2013. 